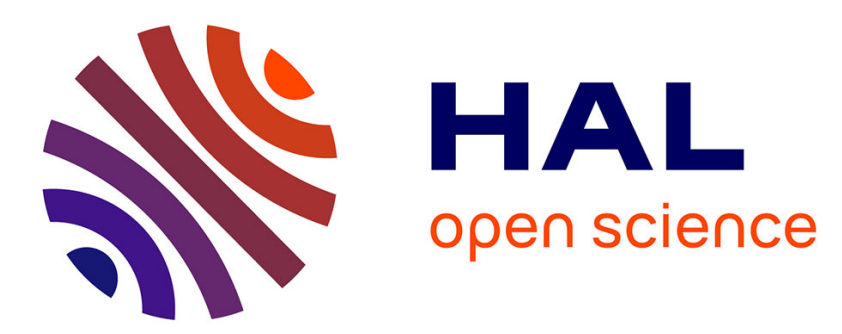

\title{
Limit distributions for coefficients of iterates of polynomials with applications to combinatorial enumerations
}

Philippe Flajolet, Andrew M. Odlyzko

\section{- To cite this version:}

Philippe Flajolet, Andrew M. Odlyzko. Limit distributions for coefficients of iterates of polynomials with applications to combinatorial enumerations. [Research Report] RR-0282, INRIA. 1984. inria00076276

\section{HAL Id: inria-00076276 \\ https://hal.inria.fr/inria-00076276}

Submitted on 24 May 2006

HAL is a multi-disciplinary open access archive for the deposit and dissemination of scientific research documents, whether they are published or not. The documents may come from teaching and research institutions in France or abroad, or from public or private research centers.
L'archive ouverte pluridisciplinaire HAL, est destinée au dépôt et à la diffusion de documents scientifiques de niveau recherche, publiés ou non, émanant des établissements d'enseignement et de recherche français ou étrangers, des laboratoires publics ou privés. 

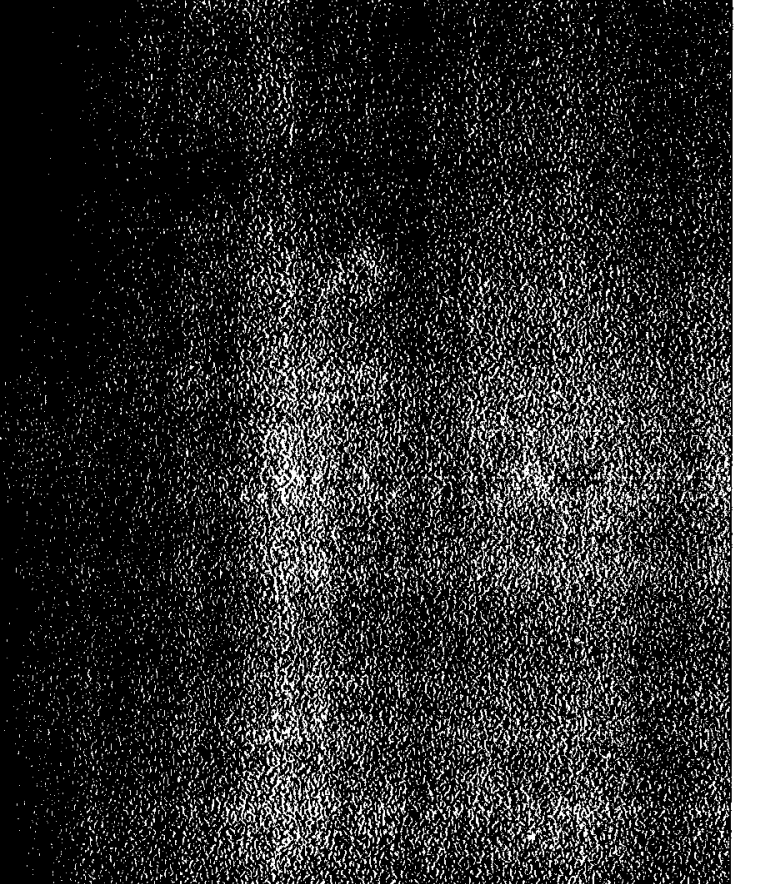

0

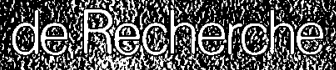
ำ. e) e. ,

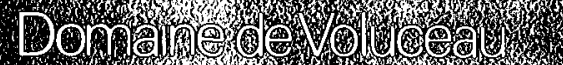
D.8.

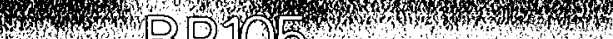

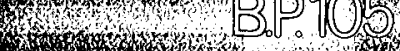


Limit Distributions for Coefficients of Iterates of Polynomials with Applications to Combinatorial Enumerations

\author{
P. Flajolet \\ INRIA \\ 78150 Rocquencourt \\ France \\ A. M. Odlyzko \\ Bell L.aporatiories \\ Murray Hill, New Jersey 079\%/4 \\ USA
}

\title{
ABSTRACT
}

This paper studies coefficients $y_{h, n}$ of sequences of polynomials

$$
y_{h}(x)=\sum_{n \geqslant 0} y_{h, n} x^{n}
$$

defined by non-linear recurrences. A typical example to which the results of this paper apply is that of the sequence

$$
B_{0}(x)=1, B_{h+1}(x)=1+x B_{h}(x)^{2} \text { for } h \geqslant 0,
$$

which arises in the study of binary trees. For a wide class of similar sequences a general distribution law for the coefficients $y_{h, n}$ as functions of $n$ with $h$ fixed is established. It follows from this law that in many interesting cases the distribution is asymptotically Gaussian near the peak. The proof relies on the saddle point method applied in a region where the polynomials grow doubly exponentially as $h \rightarrow \infty$. Applications of these results include enumerations of binary trees and 2-3 trees. Other structures of interest in computer science and combinatorics can also be studied by this method or its extensions. 
Abstract: This paper studies coefficients of sequences of polynomials defined by non-linear recurrences. A typical example to which our results apply corresponds to generating polynomials of trees of bounded height. For a wide class of recurrences a general asymptotic law for coefficients of such polynomials is established. It follows from this law that the in many interesting cases the distribution is asymptotically Gaussian near the peak. The proof relies on the saddle point method in a region where the polynomials grow doubly exponentially fast. Applications of these results include enumeration of binary trees. and 2-3 trees. Other structures of interest in computer science and combinatorics can also be studied by this method or its extensions.

Résumé: Cet article a pour objet l'etude des coefficients de polynomes définis par des recurrences non-linéaires. Un exemple typique d'application correspond aux polynomes générateurs d'arbres binaires de hauteur donnée. Pour une large classe de recurrences, on établit un résultat de distribution asymptotique concernant la distribution de ces polynomes. Il s'ensuit que ces distributions sont dans la plupart des cas d'application asymptotiquement gaussiennes dans leur région centrale. Les preuves sont obtenues par application de la méthode de col à une région où les polynomes présentent une croisssance double exponentielie. Ces résultats s'appliquent aux dénombrements d'arbres binaires, d'arbres 2-3 et à d'autres structures interessant l'informatique ou l'analyse combinatoire.

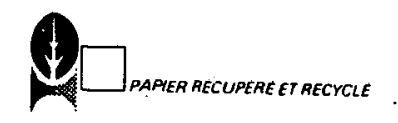




\title{
Limit Distributions for Coefficients of Iterates of Polynomials with Applications to Combinatorial Enumerations
}

\author{
P. Flajolet \\ INRIA \\ 78150 Rocquencourt \\ France \\ A. M. Odlyzko \\ Bell Laboratories \\ Murray Hill, New Jersey 07974 \\ USA
}

\section{Introduction}

In many enumerative problems in computer science and combinatorics one encounters the difficulty that no closed form formulae exist for the quantities of interest and only recurrences for generating functions are available. For example, if $B_{h, n}$ is the number of binary trees with $n$ internal nodes and height $\leqslant h$, then the generating polynomials

$$
B_{h}(z)=\sum_{n \geq 0} B_{h, n} z^{n}
$$

satisfy the recurrence [5]

$$
\left\{\begin{array}{l}
B_{h}(z)=1+z\left(B_{h-1}(z)\right)^{2} \text { for } h \geqslant 1, \\
B_{0}(z)=1
\end{array}\right.
$$

In this paper, we introduce a new method for studying coefficients of sequences of polynomials that satisfy recurrences of similar types.

We study sequences of polynomials $y_{h}(z)$, which we will refer to as PNI-sequences (for positive nonlinear iteration), with

$$
y_{h}(z)=\sum_{n} y_{h, n} z^{n}
$$

They are defined by some initial $y_{0}(z) \neq 0$ which has non-negative coefficients and a recurrence

$$
y_{h+1}(z)-P\left(z, y_{h}(z)\right), \quad h \geq 0
$$


where $\boldsymbol{P}(z, y)$ is a polynomial with non-negative coefficients,

$$
P(z, y)=\sum_{0<k<d} P_{k}(z) y^{k} \quad \text { with } p_{d}(z) \neq 0, d>1
$$

We define

$$
\begin{aligned}
& \mu=\lim _{h \rightarrow \infty} d^{-h} \operatorname{deg} y_{h}(z), \\
& \rho=\inf \left\{x: x \in \mathbb{R}^{+}, y_{h}(x) \rightarrow \infty \text { as } h \rightarrow \infty\right\} .
\end{aligned}
$$

Clearly $\mu$ and $\rho$ exist and are finite for every PNI-sequence $\left\{y_{h}(z)\right\}$ that contains non-constant polynomials. As will be explained below, it is sufficient to consider PNI-sequences which $P(z, y)$ and $y_{0}(z)$ satisfy the following conditions:

(A) $P(z, y)$ is not a monomial (i.e., $\left.P(z, y) \neq b z^{a} y^{d}\right)$.

(B) At least one of the $y_{h}, 0 \leqslant h \leqslant 2$ has the property that

$$
\left|y_{h}(z)\right|=y_{h}(1) \text { and }|z|=1 \rightarrow z=1 \text {. }
$$

We prove two main results.

Theorem 1. Suppose that $\left\{y_{h}(z)\right\}$ is a PNI-sequence that satisfies conditions $(A)$ and $(B)$, and let $\lambda_{1}$ and $\lambda_{2}$ be any real numbers that satisfy

$$
0<\lambda_{1}<\lambda_{2}<\mu
$$

Then for any integers $n$ and $h$ with

$$
\lambda_{1} \leqslant n d^{-h} \leqslant \lambda_{2}
$$

we have, uniformly in $n$ and $h$,

$$
y_{h, n}=\frac{r p_{d}(r)^{-1 /(d-1)} \exp \left(d^{h}\left(\beta(r)-r \beta^{\prime}(r) \log r\right)\right)}{d^{h / 2} \sqrt{2 \pi\left(r^{2} \beta^{\prime \prime}(r)+r \beta^{\prime}(r)\right)}}\left(1+O\left(d^{-h / 2}\right)\right)
$$

where $r$ is the unique solution in $(\rho, \infty)$ of

$$
r \beta^{\prime}(r)=n d^{-h}
$$

and $\beta(z)$ is a function which is defined on $(\rho, \infty)$ by 


$$
\beta(z)-\log y_{0}(z)+\frac{1}{d-1} \log p_{d}(z)+\sum_{j=0}^{\infty} d^{-j-1} \log \left\{\frac{y_{j+1}(z)}{p_{d}(z) y_{j}(z)^{d}}\right\},
$$

and is analytic there.

Theorem 2. Suppose that $\left.y_{h}(z)\right\}$ satisfies the conditions of Theorem 1. Let $N_{h}^{*}$ denote some $n$ for which $y_{h, n}$ is maximal. If $\rho \geqslant 1$, then

$$
\lim _{h \rightarrow \infty} d^{-h} N_{h}^{*}=0
$$

If $\rho<1$, then

$$
N_{h}^{*} \sim \beta^{\prime}(1) \dot{d^{h}} \text { as } h \rightarrow \infty
$$

and the $y_{h, n}$ are asymptotically Gaussian near the peak; for

$$
\left|n-N_{h}^{*}\right|=O\left(d^{2 h / 3}\right)
$$

we have

$$
\frac{y_{h, n}}{y_{h, N_{n}^{*}}^{*}}=\exp \left(-\frac{1}{2} \sigma^{-2} d^{-h}\left(n-N_{h}^{*}\right)^{2}\right)\left(1+O\left(d^{-2 h}\left|n-N_{h}^{*}\right|^{3}\right)\right)
$$

where

$$
\sigma^{2}=\beta^{\prime}(1)+\beta^{\prime \prime}(1)
$$

In the remainder of this section we first make some remarks about these theorems, and then discuss their connections to other work. Section 2 proves a series of auxiliary results that are at the heart of our method, and from which theorems 1 and 2 are easily deduced in Section 3. Section 4 presents some applications, possible extension, and numerical results.

Both theorems 1 and 2 give information about the coefficients of the polynomials $y_{h}(z)$ in terms of the function $\beta(z)$, which is defined by $(1.7)$ in terms of the polynomials $y_{h}(z)$. This is not circular, however, since the series in (1.7) is extremely rapidly convergent, and is determined to great accuracy by just a few initial terms. Differentiating the basic recurrence (1.2) yields a 
recurrence for $y_{h+1}^{\dot{1}}(z)$ in terms of $y_{h}(z)$ and $y_{k}^{\dot{\prime}}(z)$, and therefore the definition (1.7) of $\beta(z)$ also gives a rapid way to compute the derivatives of $\beta(z)$. As is shown by the examples in Section 4 , the approximations (1.6) and (1.9) are very accurate even for small values of $h$.

Many of the hypotheses of our theorems can be weakened. It is not essential, for example, that all the coefficients of $P(z, y)$ or of the $y_{h}(z)$ be nonnegative. What is really crucial is that the $y_{h}(z)$ should grow very rapidly as $h \rightarrow \infty$ on the positive real axis and should be relatively small elsewhere. (cf. $[6,7,14]$.) However, the appropriate growth conditions are not always easy to check, and so we have chosen to restrict our presentation to PNI-sequences, which are easy to characterize, and which are of greatest interest in computer science and combinatorics.

Condition (A) is not necessary for the success of our method. In fact, Theorem A holds far PNI-sequences which satisfy condition (B) but not condition (A), except that $\lambda_{1}$ may have to be bounded below away from 0 . However, for PNI-sequences that do not satisfy condition (A), the definition of $\beta(z)$ can be simplified. We note that if $y_{h}(z)$ is a PNI-sequence for which condition (A) fails to hold, then

$$
P(z, y)=b z^{a} y^{d}
$$

for some $b>0, a \geqslant 0$ and so

$$
y_{h}(z)=\left(b z^{\alpha}\right)^{\frac{d^{4}-1}{d-1}} y_{0}(z)^{d^{4}}
$$

and we can reduce to the study of coefficients of high powers of $y_{0}(z)$. These, however, can be investigated much more directly, without developing most of the analytic machinery of paper through use of the central limit theorem. Much stronger results can also be proved in this situation [12].

Condition (B) is very easy to check, since a polynomial

$$
y(z)=\sum_{k=0}^{m} a_{k} z^{e_{1}}, \quad 0 \leqslant e_{0}<e_{1}<\ldots<e_{m}, a_{1}, \ldots, a_{m}>0
$$

has the property that $|y(z)|-y(1)$ and $|z|-1$ imply $z-1$ if and only if 


$$
\operatorname{gcd}\left(e_{1}-e_{0}, e_{2}-e_{0}, \ldots, e_{m}-e_{0}\right)=1
$$

which holds if and only if $y(z)$ is not of the form

$$
y(z)=z^{c} y^{*}\left(z^{d}\right)
$$

for some polynomial $y^{*}(z)$ and some $d>1$. The function of condition (B) is to ensure (see Lemma 2.1) that for large $h$, the $y_{h}(z)$ are not of the form (1.10), since in that case our theorems are obviously not true. However, PNI-sequences of polynomials $y_{h}(z)$ for which each $y_{h}(z)$ is of the form

$$
z^{e_{1}} y_{h}^{*}\left(z^{d}\right)
$$

can be studied by our method by looking at the sequences $y_{h}^{*}(z)$, provided $d$ is chosen to be maximal. We also note that by the proof of Lemma 2.1 , condition (B) is equivalent to only $y_{2}(z)$ having the specified property. Lemma 2.2 shows that condition (B) cannot be weakened.

Theorems 1 and 2 are proved in Section 3, while Section 2 proves a number of auxiliary lemmas. The proofs rely on an analysis of the behavior of the polynomials $y_{h}(z)$ as $h \rightarrow \infty$, for $z \in C,|z|>\rho$. It is shown that for $z$ in a narrow strip of the form $\operatorname{Re} z>\rho+\delta,|\operatorname{Im} z|<\delta$ for some fixed $\delta>0$, the polynomials $y_{h}(z)$ exhibit doubly exponential growth:

$$
y_{h}(z)=g(z) \alpha(z)^{d^{*}}(1+o(1)) \quad \text { as } h \rightarrow \infty
$$

for certain functions $\alpha(z), g(z)$, and that the $y_{h}(z)$ are considerably smaller away from the real axis. The precise estimates we obtain enable us to determine the asymptotic behavior of the $y_{h, n}$ by expressing them as contour integrals and using the saddle point method.

The key to the success of this method is the doubly exponential growth (1.11) of the $y_{h}(z)$. Equation (1.11) generalizes the results of Aho and Sloane [2] about integer sequences satisfying nonlinear recurrences of the type

$$
x_{n+1}=x_{n}^{2}+g_{n}
$$

with $\left|g_{n}\right|<\frac{x_{n}}{4}$ for $n \geqslant n_{0}$. 
Our results are related to the immense literature on the subject of rational iteration. (See, for example, $[3,4,8]$.) Most of the papers in that area are concerned with questions of convergence of iteration. In this paper, on the other hand, we are operating almost exclusively in the region of divergence, and we concentrate on the rate and nature of divergence. In other situations, such as those of $[5,10,11,13]$, it is advantageous to study the iteration either within the convergence region or else right on the boundary between convergence and divergence. Methods similar to some of those used in those papers could also be used to obtain more information than is provided by Theorem 2 when $\rho \geqslant 1$.

\section{Proofs of Auxiliary Results}

As a first step, we prove a technical result which will enable us to show that the polynomials $y_{h}(z)$ are very small away from the positive real axis.

Lemma 2. If $\left\{y_{h}(z)\right\}$ is a PNI-sequence of polynomials that satisfies Condition (B), then for every $h \geqslant 2$ and every $r \in \mathbb{R}^{+}$,

$$
\left|y_{h}(z)\right|=y_{h}(r) \text { and }|z|=r \Rightarrow z=r
$$

Proof. Let $\left\{y_{h}(z)\right\}$ satisfy the hypotheses of the lemma. Since $y_{n}(z)$ has nonnegative coefficients, for $|z|-r, z \neq 0$, we have

$$
\left|y_{h}(z)\right|-\left|\sum_{n} y_{h, n} z^{n}\right| \leqslant \sum_{n} y_{h, n} r^{n}=y_{h}(r)
$$

and equality can hold if and only if for some $\gamma \in \mathrm{C}$ with $|\gamma|-1$,

$$
y_{h, n} z^{n}-\gamma y_{h, n} r^{n} \quad \text { for all } n \text {. }
$$

Let $u=z / r-z /|z|$. Then (2.2) is equivalent to

$$
y_{h, n} u^{n}=\gamma y_{h, n} \text { for all } n,
$$

which is equivalent to $\left|y_{h}(u)\right|=y_{h}(1)$. Thus $\left|y_{h}(z)\right| \cdots y_{h}(r)$ holds for some $z \neq r,|z|-r$ if and only if $\left|y_{h}(u)\right|=y_{h}(1)$ holds for some $u \neq 1,|u|=1$. 
Suppose now that $m \geqslant 1$ and that for some $z$ with $|z|-1$ we have $\left|y_{m}(z)\right|-y_{m}$ (1). The recurrence (1.1) implies that

$$
\left|\sum_{k=0}^{d} p_{k}(z) y_{m-1}(z)^{k}\right|-\sum_{k=0}^{d} p_{k}(1) y_{m-1}(1)^{k}
$$

Since all the coefficients of $y_{m-1}(z)$ and of the $p_{k}(z)$ are nonnegative,

$$
\begin{aligned}
\left|p_{k}(z)\right| & \leqslant p_{k}(1), 0 \leqslant k \leqslant d, \\
\left|y_{m-1}(z)\right| & \leqslant y_{m-1}(1),
\end{aligned}
$$

and so (2.3) can hold only if $\left|y_{m-1}(z)\right|=y_{m-1}$ (1). Repetition of this argument shows that if for some $z \neq 1,|z|=1$, we have $\left|y_{h}(z)\right|-y_{h}(1)$ for some $h \geqslant 2$, then $\left|y_{m}(z)\right|=y_{m}(1)$ for $0 \leqslant m \leqslant h$, and this contradicts Condition (B) and proves the lemma.

Lemma 2.1 guarantees that for PNI-sequences $\left.y_{h}(z)\right\}$ that satisfy Condition (B), $y_{h}(z)$ for $h \geqslant 2$ achieves a unique maximum on $|z|-r$ at $r$. This means, in particular, that for large $h$, $y_{h}(z)$ will not be of the form

$$
y_{h}(z)=z^{a_{k}} y_{h}^{*}\left(z^{m}\right)
$$

for some polynomials $y_{h}^{*}(u)$ and some $m>1$. The next Lemma shows that Condition (B) is in a sense best possible for our problem because if it is violated, then the polynomials $y_{h}(z)$ can be written in the form (2.4), and theorems 1 and 2 clearly cannot hold for such polynomials. The same result would not follow if we only imposed conditions on $y_{0}(z)$ and $y_{1}(z)$, as is shown by the PNIsequence defined by $y_{0}(z)-1, P(z, y)=z y+z^{3} y^{2}$. In this example $\left|y_{h}(-1)\right|-y_{h}(1)$ for $h=0,1$, but not for $h-2$, and this sequence does satisfy Condition (B).

Lemma 2.2. If $\left\{y_{h}(z)\right\}$ is a PNI-sequence of polynomials, and there is $a z \neq 1,|z|-1$, such that $\left|y_{2}(z)\right|-y_{2}(1)$, then there is an integer $r \geqslant 2$ such that for each $h \geqslant 0$.

$$
y_{h}(z)=z^{a_{s}} y_{n}^{*}\left(z^{r}\right),
$$


where the $y_{h}^{*}(n)$ are polynomials.

Proof. Suppose that $z \neq 1,|z|-1$, and $\left.y_{h}(z)\right\}$ satisfy the hypotheses of the lemma. By the arguments used in the proof of Lemma 2.1, we see that $\left|y_{1}(z)\right|=y_{1}(1)$ and $\left|y_{0}(z)\right|=y_{0}(1)$ as well.

If $y_{2, n}=0$ for $n<m$ and $y_{2, m} \neq 0$, then $\left|y_{2}(z)\right|-y_{2}(1)$ implies that

$$
\left|\sum_{n>m} y_{2, n} z^{n-m}\right|=\sum_{n \geq m} y_{2, n}
$$

Since the first term inside the absolute value sign in (2.6) is $y_{2, n}>0$, equality can hold if and only if

$$
y_{2, n} z^{n-m}=y_{2, n} \quad \text { for all } n
$$

Therefore either $y_{2, n}-0$ for all $n>m$ (i.e., $y_{2}(x)$ is a monomial) or else $z^{g}=1$ for some integer $g \geqslant 2$, and if $g$ is chosen to be minimal such that $z^{g}-1$, then $y_{2, n}=0$ if $n \not \equiv m(\bmod g)$. In the second case, if $r$ is any prime factor of $g$, then $y_{2, n}=0$ if $n \neq \equiv m(\bmod r)$. The same arguments show that each of $y_{h}(x), h=0,1$ is either a monomial or else has the property that $y_{h, n}=0$ if $n \not \equiv e_{h}(\bmod r)$, where $e_{h}$ is the smallest integer $n$ such that $y_{h, n} \not \equiv 0$. Therefore each $y_{h}(x)$, $0 \leqslant h \leqslant 2$, which is not a monomial, can be written in the form

$$
y_{h}(x)=x^{e^{4}} y_{h}^{*}\left(x^{r}\right)
$$

where $y_{h}^{*}(t)$ is a polynomial. But any monomial can obviously be written in the form $(2.8)$, so we conclude that a representation of that form exists for each $y_{h}(x), 0 \leqslant h \leqslant 2$.

Write

$$
P(x, y)=\sum_{0<i j<r} g_{i j}\left(x^{r}, y^{r}\right) x^{i} y^{j}
$$

where the $g_{i, j}(u, v)$ are polynomials with nonnegative coefficients which are uniquely determined by (2.9). Then by the basic recurrence (1.2), 


$$
y_{1}(x)=x^{e^{i} y_{1}^{*}}\left(x^{r}\right)=\sum_{i j} g_{i, j}\left(x^{r}, y_{0}(x)^{r}\right) x^{i+e_{j}} y_{0}^{*}\left(x^{r}\right)^{j}
$$

so we must have

$$
e_{1} \equiv i+e_{0} j(\bmod r)
$$

for each pair $(i, j)$ such that $g_{i j}(u, v) \neq 0$. Similarly,

$$
y_{2}(x)=x^{e_{2}} y_{2}^{*}\left(x^{r}\right)-\sum_{i, j} g_{i, j}\left(x^{r}, y_{1}(x)^{r}\right) x^{i+e_{j}} y_{1}^{*}\left(x^{r}\right)^{j}
$$

so that we must have

$$
e_{2} \equiv i+e_{1} j(\bmod r)
$$

for each pair $(i, j)$ with $g_{i, j}(u, v) \neq 0$.

Suppose first that there are two distinct pairs $(i, j)$ such that $g_{i j}(u, v) \neq 0$. Call them $\left(i_{1}, j_{1}\right)$ and $\left(i_{2}, j_{2}\right)$. Then by $(2.11)$,

$$
\begin{aligned}
& i_{1} \equiv e_{1}-e_{0} j_{1} \quad(\bmod r) \\
& i_{2} \equiv e_{1}-e_{0} j_{2} \quad(\bmod r)
\end{aligned}
$$

and if $j_{1} \equiv j_{2}(\bmod r)$, then we would have $i_{1} \equiv i_{2}(\bmod r)$, which is a contradiction, since $0 \leqslant i_{1}, i_{2}, j_{1}, j_{2} \leqslant r-1$ and $\left(i_{1}, j_{1}\right) \neq\left(i_{2}, j_{2}\right)$. Hence $j_{1} \neq j_{2}(\bmod r)$. Then by $(2.12)$ and $(2.13)$

$$
e_{2} \equiv e_{1}+\left(e_{1}-e_{0}\right) j_{1} \equiv e_{1}+\left(e_{1}-e_{0}\right) j_{2} \quad(\bmod r)
$$

which implies that $e_{1} \equiv e_{0}(\bmod r)$, since $j_{1} \not \equiv j_{2}(\bmod r)$ and $r$ is prime. But in that case

$$
e_{0} \equiv i+e_{0} j(\bmod r)
$$

for all pairs $(i, j)$ with $g_{i, j}(u, v) \neq 0$, and then an inductive argument using (2.9) shows that

$$
y_{h}(x)-x^{e} y_{n}^{*}\left(x^{\prime}\right)
$$

for all $h \geqslant 0$, and this gives the desired result.

To conclude the proof of the lemma, it only remains to consider the case that there is only one pair $(i, j)$ with $g_{i, j}(u, v) \neq 0$. But then 


$$
y_{h+1}(x)=g_{i j}\left(x^{r}, y_{h}(x)^{r}\right) x^{i} y_{h}(x)^{j}
$$

and since (2.8) holds for $0 \leqslant h \leqslant 2$, (2.14) shows that it holds for all $h \geqslant 2$ with appropriate $e_{h}$. Thus the lemma is true in this case as well.

We now derive a series of lemmas giving size estimates for the polynomials $y_{h}(z)$ which will lead to proofs of theorems 1 and 2 .

Lemma 2.3. Suppose that $\left\{y_{h}(z)\right\}$ is a PNI-sequence of polynomials and define

$$
\rho=\inf \left\{x: x \in \mathbb{R}^{+}, y_{h}(x) \rightarrow \infty \text { as } h \rightarrow \infty\right\}
$$

Then for every $\delta>0$, there exist positive constants $\gamma, \eta, \xi$ such that for $z$ in the region

$$
R(\delta)-\left\{z:|\operatorname{Im}(z)| \leqslant \eta, \rho+\delta \leqslant \operatorname{Re}(z) \leqslant \delta^{-1}\right\}
$$

we have

$$
\left|y_{h}(z)\right| \geqslant \gamma \exp \left(\xi d^{h}\right)
$$

Proof. Choose $\eta_{1}>0$ so small that $p_{d}(z)$ has no zeros in the region

$$
R_{1}=\left\{z:|\operatorname{Im}(z)| \leqslant \eta_{1}, \rho+\delta \leqslant \operatorname{Re}(z) \leqslant \delta^{-1}\right\},
$$

and let

$$
a=\min \left\{\min _{z \in R_{1}}\left|\frac{1}{2} p_{d}(z)\right| \frac{1}{2}\right\} .
$$

Then for any large enough $K_{1}$ we must have

$$
|P(z, y)|>a|y|^{d}
$$

if $z \in R_{1}$ and $|y| \geqslant K_{1}$, as can be seen from the inequality

$$
|P(z, y)|>\left.\left|p_{d}(z)\right||y|^{d}\left|1-\sum_{k=0}^{d-1}\right| \frac{p_{k}(z)}{p_{d}(z)}|| y\right|^{k-d} \mid,
$$

and the fact that the $p_{k}(z)$ are bounded for $z \in R_{1}$. 
If

$$
|y|>a^{-1 /(d-1)},
$$

then

$$
a|y|^{d}>|y|
$$

so that if

$$
K_{2}=\max \left(K_{1}, a^{-1 /(d-1)}\right)
$$

and if

$$
u_{0}=y \text { and } u_{n+1}=P\left(z, u_{n}\right) \text { for } n \geqslant 0 \text {, }
$$

then for $z \in R_{1},|y| \geqslant K_{2}$ we have

$$
u_{k} \geqslant a^{\frac{d^{*}-1}{d-1}}|y|^{d^{*}}
$$

Therefore, if $|y|$ is large enough, the $u_{k}$ exhibit doubly exponential growth.

Set

$$
K_{3}=\max \left(K_{2}, 2 a^{-1}\right),
$$

and let $h_{0}$ be such that

$$
y_{h_{e}}(\rho+\delta) \geqslant 2 K_{3}
$$

Since $y_{h_{0}}(z)$ is continuous and increasing along the positive real axis, we can find $\eta_{2}$ such that $0<\eta_{2}<\eta_{1}$ and if

$$
R_{2}=\left\{z:|\operatorname{Im}(z)| \leqslant \eta_{2}, \rho+\delta \leqslant \operatorname{Re}(z) \leqslant \delta^{-1}\right\}
$$

then

$$
\left|y_{h_{0}}(z)\right| \geqslant K_{3}
$$

for $z \in R_{2}$. But then the estimate (2.18) applies, and 


$$
\left|y_{h_{0}+k}(z)\right| \geqslant a^{\frac{d^{*}-1}{d-1}} K_{3}^{d^{*}} \geqslant\left(K_{3} a^{-1 /(d-1)}\right)^{d^{*}} \geqslant 2^{d^{*}}
$$

So that the estimate (2.16) of the lemma clearly applies for $h \geqslant h_{0}$ and $z \in R_{2}$ if we take $\gamma$ and $\xi$ small enough.

To complete the proof, it suffices to extend the estimate $(2.16)$ to all $h$. We note that if $\eta_{\epsilon}\left(0, \eta_{2}\right)$ is chosen small enough, then none of the polynomials $y_{0}(z), \ldots, y_{h_{0}-1}(z)$ will have a zero in the region $R(\delta) \subseteq R_{2}$ defined by (2.15), so that (2.16) will hold for these $y_{h}(z)$ also in that region if we take $\gamma$ small enough.

Lemma 2.4. If $\left(y_{h}(z)\right\}$ is a PNI-sequence that satisfies Condition (B), then for any $\delta, \eta>0$ there is a constant $\omega>0$ such that for $h \geqslant 2, \rho+\delta \leqslant|z| \leqslant \delta^{-1}$, and

$$
z \notin R(\delta, \eta)-\left\{z: \rho+\delta \leqslant|z|<\delta^{-1},|\operatorname{Im}(z)|<\eta\right\},
$$

we have

$$
\left|y_{h}(z)\right| \leqslant y_{h}(|z|) \exp \left(-\omega d^{h}\right)
$$

Proof. By Lemma 2.3, if $h$ is large enough, say $h \geqslant h_{0}$, and

$$
\left|y_{h}(z)\right| \leqslant y_{h}(|z|) \exp (-c)
$$

for some positive $c, e^{c} \leqslant y_{h}(|z|)^{1 / 2}$, then

$$
\begin{aligned}
\left|y_{h+1}(z)\right| & \leqslant P\left(|z| y_{h}(|z|) e^{-c}\right) \\
& \leqslant p_{d}(|z|) y_{h}(|z|)^{d} e^{-c d} \sum_{k=0}^{d} \frac{p_{d-k}(|z|)}{p_{d}(|z|)} y_{h}(|z|)^{-k} e^{c k} \\
& \leqslant y_{h+1}(|z|) e^{-c d}\left(1+O\left(e^{c} y_{h}(|z|)^{-1}\right)\right) \\
& \leqslant y_{h+1}(|z|) \exp \left(-c d+2 c \xi^{-1} d^{-h}\right) \leqslant y_{h+1}(|z|) \exp \left(-c d\left(1-d^{-h / 2}\right)\right) .
\end{aligned}
$$

By Lemma 2.1, 


$$
\left|y_{h_{0}}(z)\right| \leqslant y_{h_{0}}(|z|) e^{-r}
$$

for all $z, z \notin R(\delta), \rho+\delta \leqslant|z| \leqslant \delta^{-1}$ and some $\epsilon>0$, so that (2.20) implies

$$
\begin{aligned}
\left|y_{h_{0}+k}(z)\right| & \leqslant y_{h_{0}+k}(|z|) \exp \left(-\epsilon d^{k} \prod_{j-h_{0}}^{h_{0}+k-1}\left(1-d^{-j / 2}\right)\right) \\
& \leqslant y_{h_{0}+k}(|z|) \exp \left(-\epsilon d^{k} / 2\right)
\end{aligned}
$$

which proves the lemma for $h \geqslant h_{0}$. But the estimate (2.19) follows trivially for $2 \leqslant h \leqslant h_{0}-1$ from Lemma 2.1 if we choose $\omega$ small enough.

Lemma 2.5. If $\left\{y_{h}(z)\right\}$ is a PNI-sequence, then for any $\delta>0$ there is a $\xi>0$ such that for $z \in R(\delta)$ (defined as in Lemma 2.3 ) we have

$$
y_{h}(z)=\exp \left(d^{h} \beta(z)-\frac{1}{d-1} \log P_{d}(z)\right)\left(1+O\left(\exp \left(-\xi d^{h}\right)\right)\right)
$$

where $\beta(z)$ is defined as in Theorem 1 and is analytic in $R(\delta)$.

Proof. Since none of the $y_{h}(z)$ has a zero in $R(\delta)$, we can define

$$
v_{h}(z)-\log y_{h}(z),
$$

where for real $z$, we take the principal value of the logarithm, and for $z \in R(\delta)-\mathbb{R}$, the logarithm is determined by analytic continuation. The basic recurrence (1.2) can be written

$$
y_{h+1}(z)-p_{d}(z) y_{h}(z)^{d}\left(1+\frac{q\left(z, y_{h}(z)\right)}{p_{d}(z) y_{h}(z)^{d}}\right) \text {, }
$$

where

$$
q(z, y)-P(z, y)-p_{d}(z) y^{d}
$$

Taking logarithms of both sides of (2.24), we obtain

$$
v_{h+1}(z)=d v_{h}(z)+\log p_{d}(z)+\log \left(1+\frac{q\left(z, y_{h}(z)\right)}{p_{d}(z) y_{n}(z)^{d}}\right) .
$$


Since

$$
v_{0}(z)=\log y_{0}(z)
$$

iterating (2.26) yields

$$
v_{h}(z)=d^{h} \log y_{0}(z)+\frac{d^{h}-1}{d-1} \log p_{d}(z)+\sum_{m=1}^{h} d^{j-1} r_{h-j}(z),
$$

where

$$
r_{j}(z)-\log \left(1+\frac{q\left(z, y_{j}(z)\right)}{p_{d}(z) y_{j}(z)^{d}}\right)
$$

We now introduce the function

$$
\beta(z)=\log y_{0}(z)+\frac{1}{d-1} \log p_{d}(z)+\sum_{j=0}^{\infty} d^{-j-1} r_{j}(z)
$$

By Lemma 2.3, the $r_{j}(z)$ are bounded in $R(\delta)$, so the series in (2.29) converges and makes $\beta(z)$ an analytic function for $z \in R(\delta)$. Furthermore, (2.27) shows that

$$
v_{h}(z)=d^{h} \beta(z)-\frac{1}{d-1} \log p_{d}(z)-\sum_{j=0}^{\infty} d^{-j-1} r_{h+j}(z)
$$

and by Lemma 2.3 the last sum in $(2.30)$ is

$$
O\left(\exp \left(-\xi d^{h}\right)\right)
$$

for some $\xi>0$, which concludes the proof of the lemma.

For further reference, we note that it follows from (2.23), (2.29), and (2.30) that

$$
\beta(z)=\lim _{h \rightarrow \infty} d^{-h} v_{h}(z)-\lim _{h \rightarrow \infty} d^{-h} \log y_{h}(z)
$$

In Lemma 2.5, $\beta(z)$ was defined for $z \in R(\delta)$. However, the definition of $\beta(z)$ does not depend on $\delta$, so we conclude that $\beta(z)$ is defined and analytic in the union of all the $R(\delta)$ for $\delta>0$.

Before proceeding to the proofs of the theorems, we prove some auxiliary results about $\beta(z)$. 
Lemma 2.6. Suppose $\left(y_{n}(z)\right)$ is a PNI-sequence which satisfies conditions $(A)$ and $(B)$, and let $\mu, p$ be defined by (1.4) and (1.5), respectively. Then

$$
\left(z \beta^{\prime}(z)\right)^{\prime}>0 \text { for } z \in(\rho, \infty)
$$

and

$$
\lim _{z \rightarrow \infty} z \beta^{\prime}(z)=\mu
$$

If $P(z, y)$ is not a monomial (i.e., $\left.P(z, y) \neq b z^{a} y^{d}\right)$, then

$$
\lim _{z \rightarrow p^{+}} z \beta^{\prime}(z)=0
$$

Proof. By (2.31), for any $z \in(\rho, \infty)$, we have

$$
z \beta^{\prime}(z)=\lim _{h \rightarrow \infty} d^{-h} \frac{z y_{h}^{\prime}(z)}{y_{h}(z)} .
$$

We first observe that for any entire function $f(z) \neq 0$ with nonnegative Taylor series coefficients,

$$
f(z)-\sum_{k=0}^{\infty} f_{k} z^{k}, \quad f_{k} \geqslant 0
$$

the quotient

$$
g(z)=\frac{z f^{\prime}(z)}{f(z)}
$$

is an increasing function of $z$ for $z \in \mathbb{R}^{+}$, since computing the derivative of $g(z)$ yields

$$
z g^{\prime}(z)=z^{2} \frac{f^{\prime \prime}(z)}{f(z)}+z \frac{f^{\prime}(z)}{f(z)}-\left(\frac{z f^{\prime}(z)}{f(z)}\right)^{2},
$$

and the quantity on the right side of (2.36) is the variance of the random variable $X$ such that

$$
\operatorname{Pr}(\dot{X}-k)=\frac{f_{k} z^{k}}{f(z)}
$$

Moreover, we see that $g^{\prime}(z)=0$ is possible if and only if only one of the $f_{k}$ is $\neq 0$.

Next, we prove that if $f(z)-f_{1}(z)+f_{2}(z)$, where $f_{1}(z)$ and $f_{2}(z)$ are both nonzero entire functions with nonnegative Taylor coefficients, 


$$
f_{i}(z)=\sum_{k=0}^{\infty} f_{i, k} z^{k}, \quad i=1,2
$$

then

$$
z\left(\frac{z f^{\prime}(z)}{f(z)}\right)^{\prime} \geqslant \frac{f_{1}(z)}{f(z)} \cdot z\left(\frac{z f_{1}^{\prime}(z)}{f_{1}(z)}\right)^{\prime}
$$

for any $z \in \mathbb{R}^{+}$. To see this, note that by the preceding paragraph, the quantity on the left side of (2.37) is the variance of the random variable $X$ such that

$$
\operatorname{Pr}(X-k)=\frac{f_{k} z^{k}}{f(z)}
$$

But $X$ is a mixture of the random variables $X_{1}$ and $X_{2}$, where

$$
\operatorname{Pr}\left(X_{i}=k\right)=\frac{f_{i, k} z^{k}}{f_{i}(k)}
$$

with weights $f_{i}(z) / f(z)$. (A mixture $\lambda Y_{1}+(1-\lambda) Y_{2}$ of random variables $Y_{1}$ and $Y_{2}$ with weights $\lambda$ and $1-\lambda$ corresponds to choosing $Y_{1}$ with probability $\lambda$ and $Y_{2}$ with probability $1-\lambda$.) Thus to prove (2.37), it will suffice to show that if $Y_{1}$ and $Y_{2}$ are any real-valued random variables, and $\lambda \in[0,1]$, then

$$
\operatorname{Var}\left(\lambda Y_{1}+(1-\lambda) Y_{2}\right) \geqslant \lambda \operatorname{Var}\left(Y_{1}\right)+(1-\lambda) \operatorname{Var}\left(Y_{2}\right)
$$

If $F_{i}$ denotes the distribution function of $X_{i}$, then (2.38) is equivalent to

$$
\begin{aligned}
& \lambda \int x^{2} d F_{1}+(1-\lambda) \int x^{2} d F_{2}-\left(\lambda \int x d F_{1}+(1-\lambda) \int x d F_{2}\right)^{2} \\
& \geqslant \lambda \int x^{2} d F_{1}-\lambda\left(\int x d F_{1}\right)^{2}+(1-\lambda) \int x^{2} d F_{2}-(1-\lambda)\left(\int x d F_{2}\right)^{2},
\end{aligned}
$$

which is easily seen to hold. This completes the proof of (2.37).

We now apply (2.37) is with

$$
f_{1}(z)=p_{d}(z) y_{h}(z)^{d}, f_{2}(z)=y_{h+1}(z)-f_{1}(z)-P\left(z, y_{h}(z)\right)-p_{d}(z) y_{h}(z)^{d} .
$$

We discover 


$$
\begin{aligned}
& z\left(\frac{z y_{h+1}(z)}{y_{h+1}(z)}\right)^{\prime} \geqslant \frac{p_{d}(z) y_{h}(z)^{d}}{y_{h+1}(z)} \cdot z \cdot\left(\frac{z p_{d}(z)}{p_{d}(z)}+d \frac{z y_{h}(z)}{y_{h}(z)}\right)^{\prime} \\
& \geqslant d \frac{p_{d}(z) y_{h}(z)^{d}}{y_{h+1}(z)} \cdot z\left(\frac{z y_{h}^{\prime}(z)}{y_{n}(z)}\right)^{\prime} \text {. }
\end{aligned}
$$

If we iterate this inequality, we obtain

$$
z\left(\frac{z y_{h+1}^{\prime}(z)}{y_{h+1}(z)}\right)^{\prime} \geqslant d^{h-2} z\left(\frac{z y_{2}(z)}{y_{2}(z)}\right)^{\prime} \cdot \prod_{j=2}^{h} \frac{p_{d}(z) y_{j}(z)^{d}}{y_{j+1}(z)} .
$$

Now Lemma 2.3 implies that the product

$$
\prod_{j=2}^{\infty} \frac{p_{d}(z) y_{j}(z)^{d}}{y_{j+1}(z)}
$$

converges to a number $b=b(z)>0$, and since each factor is $\leqslant 1$, we deduce from (2.39) that

$$
d^{-h} z\left(\frac{z y_{h}^{\prime}(z)}{y_{h}(z)}\right)^{\prime} \geqslant d^{-1} b z\left(\frac{z y_{2}^{\prime}(z)}{y_{2}(z)}\right)^{\prime}
$$

and the last factor on the right side in (2.40) is $>0$ by Condition (B). Since $z\left(z \beta^{\prime}(z)\right)^{\prime}$ is the limit of the left side of (2.40) as $h \rightarrow \infty$, we obtain the claim (2.32) of the lemma.

To prove (2.33), we note that if $f(z)$ is any polynomial with nonnegative coefficients, then

$$
z f^{\prime}(z) \leqslant \operatorname{deg}(f(z)) \cdot f(z), \quad z \in \mathbb{R}^{+},
$$

and so

$$
z \beta^{\prime}(z) \leqslant \lim _{h \rightarrow \infty} d^{-h} \operatorname{deg} y_{h}(z)=\mu .
$$

To complete the proof of (2.33), note that for $h \geqslant h_{0}$,

$$
\operatorname{deg} y_{h+1}(z)-d \operatorname{deg} y_{h}(z)+\operatorname{deg} p_{d}(z)
$$

and so

$$
\operatorname{deg} y_{h_{0}+k}(z)=d^{k} \operatorname{deg} y_{h_{0}}(z)+\frac{d^{k}-1}{d-1} \operatorname{deg} p_{d}(z)
$$

Next, note that for $z \in \mathbb{R}^{+}$, 


$$
y_{h+1}^{\prime}(z) \geqslant d p_{d}(z) y_{h}(z)^{d-1} y_{h}^{\prime}(z)
$$

and so

$$
\frac{y_{h+1}^{\dot{1}}(z)}{y_{h+1}(z)} \geqslant d \frac{y_{h}^{\dot{\prime}}(z)}{y_{h}(z)}\left(1-\gamma e^{-\xi d^{d}}\right)
$$

for some $\gamma, \xi>0$, where this holds uniformly for all $h \geqslant 1$ and all $z \in(\rho+1, \infty)$ by Lemma 2.3 (applied with any $\delta<1$ such that $R(\delta) \neq \phi$ ) and the fact that each of the $y_{h}(z)$ is increasing on $\mathbb{R}^{+}$. Therefore for any $\epsilon>0$, if we choose $h_{1}$ such that

$$
\prod_{h=h_{1}}^{\infty}\left(1-\gamma e^{-\xi d^{\star}}\right)>1-\epsilon / 2,
$$

then for any $z \in(\rho+1, \infty)$ and any $h \geqslant h_{1}$ we will have

$$
z \beta^{\prime}(z) \geqslant d^{-h} \frac{z y_{h}^{\prime}(z)}{y_{h}(z)}(1-\epsilon / 2)
$$

If we now choose $h_{2} \geqslant \max \left(h_{0}, h_{1}\right)$, and $z$ so large that

$$
\frac{z y_{h_{2}}(z)}{y_{n_{2}}(z)} \geqslant(1-\epsilon / 10) \operatorname{deg} y_{h_{2}}(z)
$$

then by (2.43) we will have

$$
z \beta^{\prime}(z) \geqslant(1-\epsilon) d^{-h_{2}} \operatorname{deg} y_{h_{2}}(z) .
$$

Since by (2.42)

$$
d^{-h_{1}} \operatorname{deg} y_{h_{2}}(z)-\lim _{h \rightarrow \infty} d^{-h} \operatorname{deg} y_{h}(z)-\mu
$$

this together with (2.41) proves (2.33).

To complete the proof of the lemma, we need to prove $(2.34)$ when $P(z, y)$ is not a monomial. Define 


$$
\begin{aligned}
& t_{h}(z)=d^{-k} \frac{y_{h}^{\dot{h}}(z)}{y_{h}(z)}, \\
& a_{h}(z)=\frac{\sum_{k} p_{k}^{\dot{y}}(z) y_{h}(z)^{k}}{\sum_{k} p_{k}(z) y_{h}(z)^{k}}, \\
& b_{h}(z)=\frac{\sum_{k} \frac{k}{d} p_{k}(z) y_{h}(z)^{k}}{\sum_{k} p_{k}(z) y_{h}(z)^{k}} .
\end{aligned}
$$

Then the recurrence (1.2) gives

$$
t_{h+1}(z)=d^{-h-1} a_{h}(z)+b_{h}(z) t_{h}(z) .
$$

If $E=\max \left\{\operatorname{deg} p_{k}(z)\right\}$, then comparison of terms in the numerators and denominators of (2.45) and (2.46) shows that for any $z \in \mathbb{R}^{+}$,

$$
\begin{aligned}
& 0 \leqslant a_{h}(z) \leqslant E z^{-1}, \\
& 0 \leqslant b_{h}(z) \leqslant 1 .
\end{aligned}
$$

Hence

$$
t_{h+1}(z) \leqslant t_{h}(z)+O\left(d^{-h} z^{-1}\right)
$$

and therefore

$$
t_{h+m}(z) \leqslant t_{h}(z)+C d^{-h} z^{-1}
$$

for all $m \in Z^{+}$and some $C>0$.

Let us first suppose that $\rho \neq 0$. We show that in this case $y_{h}(\rho)$ is bounded as $h \rightarrow \infty$. To see this, note that for every $r \in \mathbb{R}^{+}$there is a $Y(r)>0$ such that $P(z, y)>2 y$ for $z \geqslant r, y \geqslant Y(r)$. Now if $y_{h}(\rho)$ is unbounded as $h \rightarrow \infty$, then by continuity we must have $y_{k}\left(\rho^{\prime}\right)>Y(\rho / 2)$ some large $k$ and for some $\rho^{\prime} \in(\rho / 2, \rho)$, and then $y_{h}\left(\rho^{\prime}\right)$ is also unbounded as $h \rightarrow \infty$ by the argument above, which contradicts the definition of $\rho$.

Since $y_{h}(\rho)$ is bounded and $P(x, y)$ is not a monomial, we see from $(2.46)$ that there is some $B<1$ such that 


$$
b_{n}(\rho) \leqslant B, \quad h \geqslant 0 .
$$

Hence

$$
t_{h+1}(\rho) \leqslant B t_{h}(\rho)+O\left(d^{-h}\right)
$$

Since the $t_{h}(\rho)$ are bounded as $h \rightarrow \infty$, as is shown by (2.49), we find by iterating (2.50) that for some $C_{1}>0$

$$
t_{2 h}(\rho) \leqslant \ddot{C}_{1}\left(B^{h}+d^{-h}\right), \quad t_{2 h+1} \leqslant C_{1}\left(B^{h}+d^{-h}\right)
$$

Hence $t_{h}(\rho) \rightarrow 0$ as $h \rightarrow \infty$. Given $\mathrm{c}>0$, let us choose $h_{0}$ so that

$$
C d^{-h_{0}}+C_{1}\left(B^{h_{0}}+d^{-h_{0}}\right)<\epsilon / 4
$$

Then there is a $\delta>0$ such that

$$
t_{2 h_{0}}(z) \leqslant \epsilon / 2
$$

for $\rho \leqslant z \leqslant \rho+\delta$. But then (2.49) and (2.52) imply that

$$
t_{h}(z) \leqslant \epsilon
$$

for all $h \geqslant 2 h_{0}$ and $z \in[\rho, \rho+\delta]$, which implies that $\beta^{\prime}(z) \leqslant \epsilon$ for $z$ in that interval. Since this holds for every $\epsilon>0$, we must have $\beta^{\prime}(z) \rightarrow 0$ as $z \rightarrow \rho$.

To complete the proof of the lemma, we need to prove $(2.34)$ when $\rho=0$. We first observe that it will suffice to show that

$$
\lim _{h \rightarrow \infty} \lim _{z \rightarrow 0^{+}} z t_{h}(z)=0
$$

To see this, note that if $(2.53)$ holds, then for any $\epsilon>0$ we can find $h_{0}$ and $\delta>0$ such that for $z \in(0, \delta)$,

$$
z t_{h_{0}}(z) \leqslant \epsilon / 4, \quad C d^{-h_{0}} \leqslant \epsilon / 4 .
$$

But then (2.49) shows that

$$
z t_{h_{0}+m}(z) \leqslant \epsilon, \quad m \in Z^{+}, \quad z \in(0, \delta)
$$


which proves the claim.

Suppose now that $\rho=0$ and that $y_{h}(0)-0$ for all large $h$. If we write

$$
y_{h}(z)=z^{v^{*}} y_{h}^{*}(z)
$$

where $y_{h}^{*}(z)$ is a polynomial with $y_{h}^{*}(0) \neq 0$, then

$$
\lim _{z \rightarrow 0^{+}} \frac{z y_{n}^{\prime}(z)}{y_{h}(z)}=v_{h}
$$

But $P(x, y)$ is not a monomial, so $v_{n+1} \leqslant(d-1) v_{h}$, and therefore

$$
\lim _{z \rightarrow 0^{+}} z t_{n}(z) \leqslant\left(1-d^{-1}\right)^{h}
$$

which proves $(2.53)$ in this case. On the other hand, if $y_{h}(0) \neq 0$, then

$$
\lim _{z \rightarrow 0} \frac{z y_{h}(z)}{y_{h}(z)}=0
$$

and (2.53) again holds. This finally concludes the proof of the lemma.

\section{Proofs of the Theorems}

We now use the results of Section 2 to prove Theorem 1. Suppose that all the hypotheses of that theorem are satisfied. We use the Cauchy integral representation

$$
y_{h, n}=\frac{1}{2 \pi i} \int_{\Gamma} y_{h}(z) z^{-n-1} d z
$$

which is valid for any simple closed curve with the origin in its interior.

Let

$$
\lambda=\frac{n}{d^{h}}
$$

so that $\lambda_{1} \leqslant \lambda \leqslant \lambda_{2}$. We choose for $\Gamma$ the circle centered at the origin of radius $r$, where

$$
r \beta^{\prime}(r)=\lambda
$$


Since $z \beta^{\prime}(z)$ is strictly increasing from 0 to $\mu$ between $z-\rho$ and $z=\infty$ by Lemma 2.6, Eq. (3.3) defines $r$ uniquely and shows that for $\lambda \in\left[\lambda_{1}, \lambda_{2}\right], r \in\left[r_{1}, r_{2}\right]$, where $\rho<r_{1}<r_{2}<\infty$. The choice of the above contour is inspired by the fact that $r$ satisfying (3.3) is an approximate saddle point of the integrand in (3.1).

By Lemma 2.5, we find that there is a constant $\theta_{0}>0$ such that $\beta(z)$ is analytic in the region

$$
r_{1} \leqslant|z| \leqslant r_{2},-\theta_{0} \leqslant \operatorname{Arg}(z) \leqslant \theta_{0}
$$

In that region we have the expansion

$$
\operatorname{Re} \beta\left(r e^{i \theta}\right)=\beta(r)-\frac{1}{2} \theta^{2}\left(r^{2} \beta^{\prime \prime}(r)+r \beta^{\prime}(r)\right)+O\left(\theta^{4}\right)
$$

and, by taking $\theta_{0}$ small enough, we can ensure that

$$
\operatorname{Re} \beta\left(r e^{i \theta}\right) \leqslant \beta(r)-\frac{1}{4} \theta^{2}\left(r^{2} \beta^{\prime \prime}(r)+r \beta^{\prime}(r)\right)
$$

If $\Gamma_{1}$ denotes the section of the circle $z-r e^{i \theta}$ with $\theta_{0} \leqslant \theta \leqslant 2 \pi-\theta_{0}$, then by lemmas 2.4 and 2.5 ,

$$
\frac{1}{2 \pi i} \int_{\Gamma_{1}} y_{h}(z) z^{-n-1} d z=O\left(r^{-n} \exp \left(d^{h}(\beta(r)-w)\right)\right)
$$

where $w>0$ depends only on $r_{1}, r_{2}$, and $\theta_{0}$. If $\Gamma_{2}$ denotes the section of this same circle with $-\theta_{0} \leqslant \theta \leqslant \theta_{0}$, then Lemma 2.5 implies that

$$
\begin{aligned}
\frac{1}{2 \pi i} \int_{\Gamma_{2}} y_{h}(z) z^{-n-1} d z & =\frac{1}{2 \pi i} \int_{\Gamma_{2}} p_{d}(z)^{-1 /(d-1)} \exp \left(d^{h} \beta(z)\right) z^{-n-1} d z \\
& +O\left(r^{-n} \exp \left(d^{n}\left(\beta(r)-w^{\prime}\right)\right)\right)
\end{aligned}
$$

where $w^{\prime}>0$ again depends only on $r_{1}, r_{2}$, and $\theta_{0}$. To estimate the integral on the right side of (3.6), we write

$$
\Gamma_{2}-\Gamma_{3} \cup \Gamma_{4},
$$

where 


$$
\Gamma_{3}-\left\{r e^{\prime 0}:-\theta_{1} \leqslant \theta \leqslant \theta_{1}, \theta_{1}=h d^{-h / 2}\right\}
$$

On $\Gamma_{4}=\Gamma_{2} \mid \Gamma_{3},(3.5)$ yields

$$
\operatorname{Re} \beta\left(r e^{i \theta}\right) \leqslant \beta(r)-w^{\prime \prime} h^{2} d^{-h}
$$

for some $w^{\prime \prime}>0$ which depends only on $r_{1}$ and $r_{2}$, and so

$$
\frac{1}{2 \pi i} \int_{\Gamma_{4}} p_{d}(z)^{-1 /(d-1)} \exp \left(d^{h} \beta(z)\right) z^{-n-1} d z=O\left(r^{-n} \exp \left(d^{h} \beta(r)-w^{\prime \prime} h^{2}\right)\right)
$$

Finally, if

$$
J=\frac{1}{2 \pi i} \int_{\Gamma_{1}} p_{d}(t)^{-1 /(d-1)} \exp \left(d^{h} \beta(z)\right) z^{-n-1} d z
$$

then

$$
J=\frac{1}{2 \pi} \int_{-\theta_{1}}^{\theta_{1}} p_{d}\left(r e^{i \theta-1 /(d-1)} \exp \left(d^{h} \beta\left(r e^{i \theta}\right)-n \log r-n i \theta\right) d \theta\right.
$$

But (3.2), (3.4), and

$$
p_{d}\left(r e^{i \theta}\right)^{-1 /(d-1)}=p_{d}(r)^{-1 /(d-1)}(1+O(|\theta|))
$$

imply that

$$
\begin{aligned}
J & =(2 \pi)^{-1} A(r, n) \int_{\theta_{1}}^{\theta_{1}} \exp \left(-\frac{1}{2} d^{h}\left(r^{2} \beta^{\prime \prime}(r)+r \beta^{\prime}(r)\right) \theta^{2}\right) \cdot\left(1+O(|\theta|)+O\left(d^{h}|\theta|^{3}\right)\right) d|\theta| \\
& =A(r, n) d^{-h / 2}\left(2 \pi\left(r^{2} \beta^{\prime \prime}(r)+r \beta^{\prime}(r)\right)^{-1 / 2} \cdot\left(1+O\left(d^{-h / 2}\right)\right),\right.
\end{aligned}
$$

where

$$
A(r, n)-p_{d}(r)^{-1 /(d-1)} \exp \left(d^{h} \beta(r)-n \log r\right)
$$

which together with the previous estimates proves Theorem 1.

From Theorem 1, we see that the largest values of $y_{h, n}$ when $n$ varies correspond to values of $n$ (defined by (3.3)) which maximize 


$$
g(r)-\beta(r)-r \beta^{\prime}(r) \log r
$$

Now

$$
g^{\prime}(r)=-\left(\beta^{\prime}(r)+r \beta^{\prime \prime}(r)\right) \log r
$$

and since $\beta^{\prime}(r)+r \beta^{\prime \prime}(r)>0$ for $r>\rho$ by Lemma $2.6, g^{\prime}(r)$ will have a unique maximum at $r=1$ if $\rho<1$, and will be $<0$ in $(\rho, \infty)$ if $\rho \geqslant 1$. To complete the proof of Theorem 2, we need to consider $\rho<1$ and study the distribution of $y_{h, n}$ for $r$ near the peak. Define

$$
n_{0}=n_{0}(h)=\beta^{\prime}(1) d^{h},
$$

and set

$$
x=\left(n-n_{0}\right) d^{-h / 2} .
$$

We will consider

$$
|x| \leqslant d^{h / 6}
$$

If $r$ is defined by

$$
r \beta^{\prime}(r)=n d^{-h}
$$

then

$$
\begin{aligned}
\left(n-n_{0}\right) d^{-h} & =r \beta^{\prime}(r)-\beta^{\prime}(1) \\
& =(r-1) \sigma^{2}+O\left((r-1)^{2}\right),
\end{aligned}
$$

where

$$
\sigma^{2}=\beta^{\prime}(1)+\beta^{\prime \prime}(1)
$$

Hence we have

$$
r-1=x d^{-h / 2} \sigma^{2}+O\left(x^{2} d^{-h}\right)
$$

Expanding the quantities that occur in the statement of Theorem 1 in a similar way, we obtain Theorem 2. 


\section{Applications and Extensions}

The problem that originally led to our investigation was that of estimating $B_{h, n}$, the number of binary trees of height $\leqslant h$ and having $n$ internal nodes. The recurrence for the generating polynomials is given in the first paragraph of this paper. It is easy to see that $\rho=0$ and $\mu-1$. Theorems 1 and 2 imply that for large but fixed $h, B_{h, n}$ is maximized for

$$
n-2^{h} 0.628968 \ldots
$$

and that its maximum value is asymptotic to

$$
2^{-h / 2} \cdot \exp \left(2^{h} \cdot 0.407354 \ldots\right) \cdot 0.685517 \ldots
$$

For $h=9, B_{9, n}$ is maximized for $n=322$, as predicted by (4.1), and the value of $B_{9,322}$ differs from that predicted by $(4.2)$ by less than $0.05 \%$, which demonstrates how accurate the asymptotic approximations of our theorems are. Fig. 1 presents a graph of the function $\beta(r)$, defined as in Theorem 1. Fig. 2 shows a graph of the function

$$
f(\lambda)=\beta(r)-r \beta^{\prime}(r) \log r
$$

where $r$ is determined by $0<r<1$, and $r$ is determined by

$$
r \beta^{\prime}(r)=\lambda
$$

This function dominates the behavior of $B_{h, n}$, so that if $h \rightarrow \infty$ and

$$
n \sim \lambda 2^{h} \text { as } h \rightarrow \infty,
$$

then

$$
\lim _{h \rightarrow \infty} 2^{-h} \log B_{h, n}-f(\lambda)
$$

There are many enumerative problems which involve nonlinear iterations of polynomial generating functions, but which are not covered by our theorems. As an example, enumeration of AVL-trees (also known as height-balanced binary trees $[1,9]$ ) leads [11] to the polynomial sequence defined by 


$$
\begin{aligned}
y_{0}(z) & =z, \quad y_{1}(z)-z^{2}, \\
y_{h+1}(z) & =y_{h}(z)\left(y_{h}(z)+2 y_{h-1}(z)\right) \text { for } h \geqslant 1 .
\end{aligned}
$$

Since $y_{h+1}(z)$ depends on $\dot{y}_{k-1}(z)$ as well as on $y_{h}(z)$, our results do not apply directly. However, it should be possible to use the methods of this paper to prove results analogous to theorems 1 and 2 for these polynomials, as well as for many other sequences satisfying similar recurrences.

It is also possible to use the methods of this paper to study recurrences such as (1.2) where the $y_{h}(z)$ are entire functions with nonnegative coefficients and where $P(z, y)$ might also not be a polynomial. However, in many cases it is simpler to use the results of $[6,7,14]$.

Finally, we mention that it should be possible to use our methods to study multivariate polynomials satisfying nonlinear recurrences. Such polynomials occur, for example, in studies of 2,3-trees [15], where one is interested in the coefficients of the polynomials $A_{h}(x, y)$ defined by $A_{0}(x, y)=1$, and

$$
A_{h+1}(x, y)=x y A_{h}(x, y)^{2}+x y^{2} A_{h}(x, y)^{3} \text { for } h \geqslant 0 .
$$

By applying our theorems to the sequences $A_{n}(x, 1)$ and $A_{h}(1, y)$, we can obtain more precise information than is provided by [15], but it might be interesting to obtain estimates for the full distribution of the coefficients of the $A_{h}(x, y)$. 


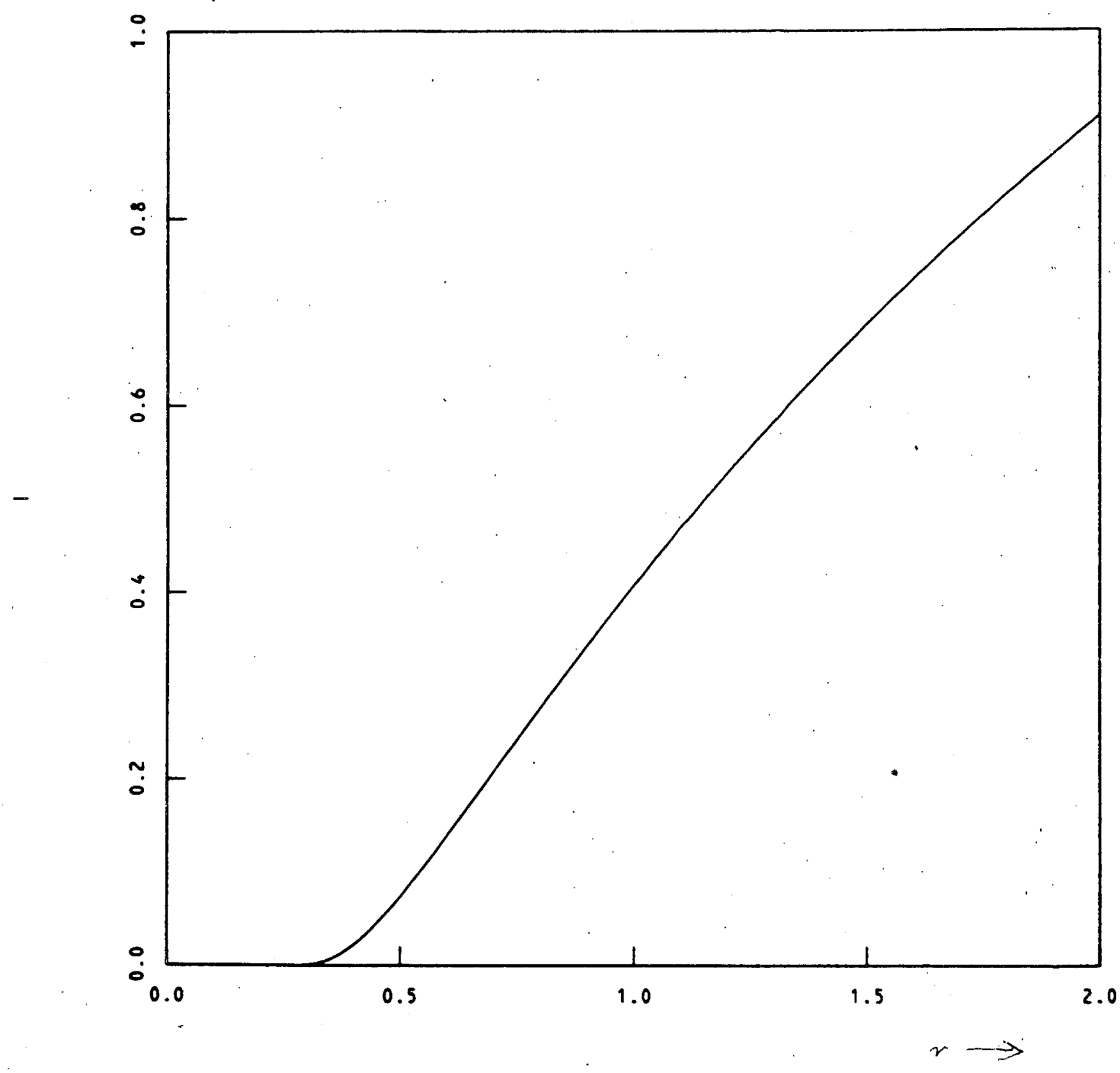

Fig. 1. The function $\beta(r)$ for binary trees. 


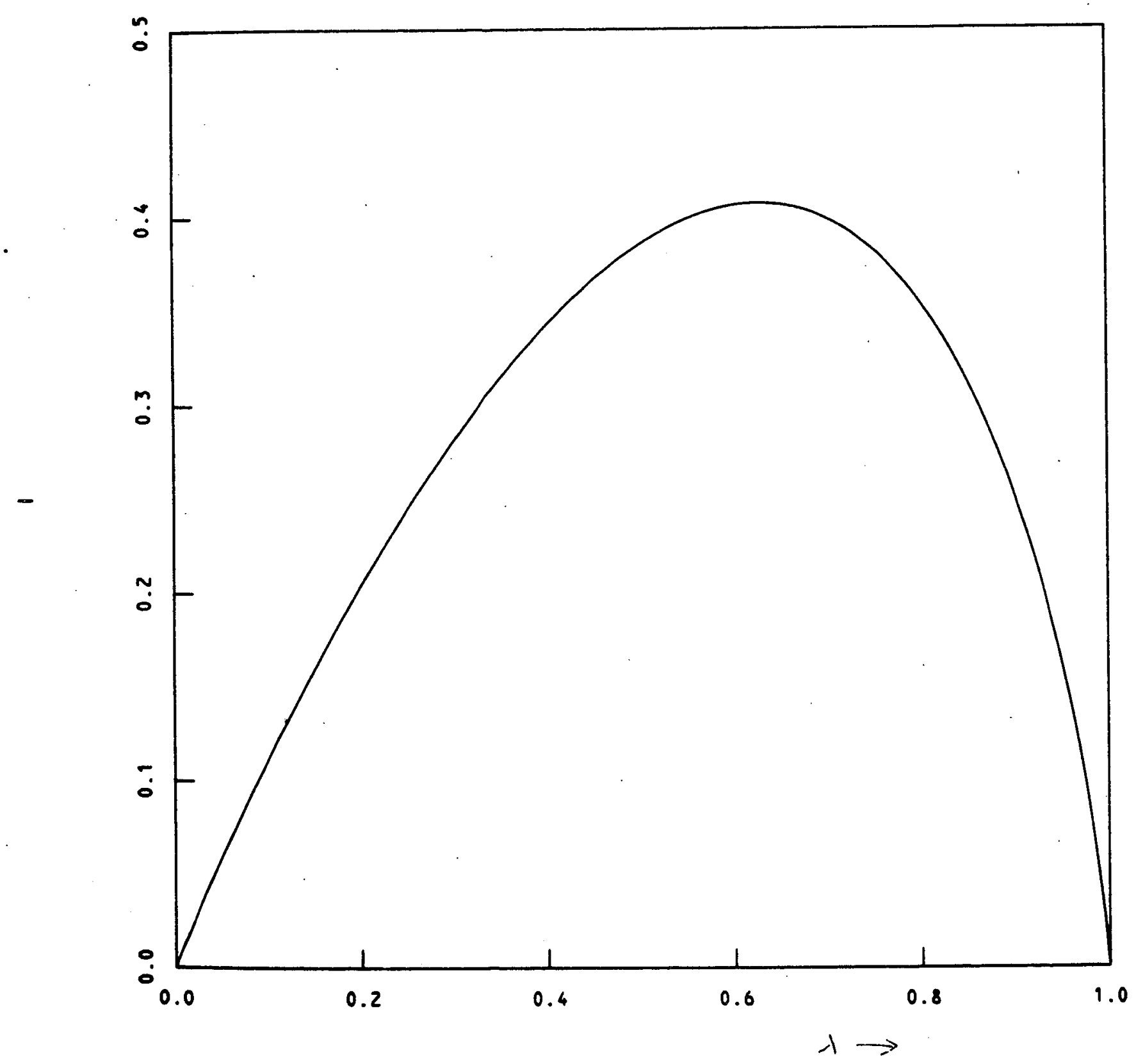

Fig. 2. The function $f(\lambda)$, which equals the limit of $2^{-h} \log B_{h, n}$ as $h, n \rightarrow \infty$ with $n-\lambda 2^{h}$. 


\section{REFERENCES.}

[1] A. V. Aho, J. E. Hopcroft, and J. D. Ullman, The Design and Analysis of Computer Algorithms, Addison-Wesley, 1974.

[2] A. V. Aho and N. J. A. Sloane, Some doubly exponential sequences, Fibonacci Quart. 11 (1973), 429-437.

[3] H. Brolin, Invariant sets under iteration of rational functions, Arkiv för Mat. 6 (1965), 103144.

[4] P. Fatou, Memoire sur les équations fonctionnelles, Bull. Soc. Math. France 47 (1919), 161$271 ; 48(1920), 33-94$ and 208-314.

[5] P. Flajolet and A. M. Odlyzko, The average height of binary trees and other simple trees, J. Computer Syst. Sci., 25 (1982), 171-213.

[6] B. Harris and L. Schoenfeld, Asymptotic expansions for the coefficients of analytic functions, Illinois J. Math. 12 (1968), 264-277.

[7] W. K. Hayman, A generalization of Stirling's formula, J. reine angew. Math. 196 (1956), $67-95$.

[8] G. Julia, Mémoire sur l'iteration des fonctions rationnelles, J. Math. Pures Appl. (8) I (1918), 47-245.

[9] D. E. Knuth, The Art of Computer Programming. Vol. I: Fundamental Algorithms, Addison-Wesley, 1968.

[10] A. M. Odlyzko, Periodic oscillations of coefficients of power series that satisfy functional equations, Adv. Math. 44 (1982), 180-205.

[11] A. M. Odlyzko, Enumeration of AVL-trees, manuscript in preparation.

[12] A. M. Odlyzko and L. B. Richmond, On the unimodality of high convolutions of discrete 
distributions, to be published.

[13] A. M. Odlyzko and L. B. Richmond, manuscript in preparation.

[14] A. M. Odlyzko and L. B. Richmond, Asymptotic expansions for the coefficients of analytic generating functions, Aequationes Math., to appear.

[15] E. M. Reingold, A note on 3-2 trees, Fibonacci Quart. 17 (1979), 151-157.

Imprimé en France

l'Institut National de Recherche en Informatique et en Automatique 
